\title{
Identification of Hypoglycemia-dependent Endothelial Nitric Oxide Synthase 0-GIcNAcylation Sites and Regulation of O-GlcNAcylation and Nitric Oxide Production by Hypoglycemia
}

an he ( $\square$ heanaichirou@163.com )

Chongqing Medical University First Affiliated Hospital

Shupeng $\mathrm{Hu}$

Chongqing Medical University First Affiliated Hospital

Qiangzhong Pi

Chongqing Medical University First Affiliated Hospital

Yongzheng Guo

Chongqing Medical University First Affiliated Hospital

\section{Yang Long}

Chongqing Medical University First Affiliated Hospital

Suxin Luo

Chongqing Medical University First Affiliated Hospital

Yong Xia

Chongqing Medical University First Affiliated Hospital

Original investigation

Keywords: Hypoglycemia, eNOS O-GIcNAcylation, AMPK, OGT, novel O-GIcNAcylation site

Posted Date: April 21st, 2020

DOI: https://doi.org/10.21203/rs.3.rs-22780/v1

License: (c) (1) This work is licensed under a Creative Commons Attribution 4.0 International License. Read Full License 


\section{Abstract}

\section{Background}

0-GlcNAcylation, an energy-sensitive post-translational modification, plays a major role in endothelial nitric oxide synthase (eNOS) activity regulation. However, the effect of hypoglycemia on eNOS 0GIcNAcylation and whether eNOS exists the novel O-GIcNAcylation sites under hypoglycemia is unknown. Hence, we endeavored to determine the effects of hypoglycemia on eNOS O-GIcNAcylation and the novel O-GIcNAcylation sites of eNOS.

\section{Method}

Bovine aortic endothelial cells (BAECs) and Sprague-Dawley rats were treated by hypoglycemia, and using immunoblotting to measure their eNOS O-GIcNAcylation. eNOS and transfected eNOS were purified by pull-down assay and immunoprecipitation respectively. Novel O-GlcNAcylation sites of eNOS were predicted by HPLC-MS and MS/MS Ion, and determined by immunoblotting. eNOS activity were detected by Elisa and isotope labelling method.

\section{Results}

In BAECs and rats' thoracic aorta, hypoglycemia-associated activation of eNOS was accompanied by an increase in O-GlcNAcylation and had no effect on O-linked serine phosphorylation at residue 1179/1177. Changes in this post-translational modification were associated with increased O-GlcNAc transferase (OGT) activity, and were reversed by AMPK knockdown. Immunoblot analysis of cells expressing Histagged wild-type human eNOS and human eNOS carrying a mutation at the Ser1177 phosphorylation site confirmed the increase in 0-GlcNAcylation in response to hypoglycemia. The observed increase in 0 GIcNAcylation indicated that eNOS contains novel O-GIcNAcylation sites that are activated by hypoglycemia. Immunoblot analysis of cells expressing His-tagged human eNOS carrying a mutation at Ser738 and Ser867 confirmed the increase in 0-GlcNAcylation in response to hypoglycemia. Contrastingly, in His-tagged human eNOS carrying a mutation at Thr866, 0-GlcNAcylation was unaffected by hypoglycemia. Differences among culture conditions were identified using two-way analysis of variance (ANOVA), one-way ANOVA, or unpaired Student's $t$-test.

\section{Conclusions}

Hypoglycemia increases eNOS O-GIcNAcylation and activity, potentially via AMPK-OGT pathway, thereby showing the Thr866 as a novel O-GlcNAcylation site involved in hypoglycemia-mediated eNOS activation.

\section{Background}

eNOS plays an important role in regulating the cardiovascular system. eNOS also functions in the synthesis of nitric oxide (NO), an endogenous vasodilator, thus playing a key role in vasodilatation (1). Endothelial NO also contributes to vessel homeostasis by regulating cell growth, platelet aggregation, and 
the binding of leukocytes to endothelial cells (2-4). Endothelium-dependent relaxation is dysregulated in both micro-circulation and macro-circulation during acute hypoglycemia in both normal subjects and patients with diabetes, suggesting that eNOS activity may be affected by hypoglycemia $(5,6)$. eNOS activity is subject to several overlapping modes of post-translation modifications; phosphorylation and OGIcNAcylation are two major post-translation modifications of eNOS that provide mechanisms for the dynamic stimulation and inhibition of enzymatic activity (7).

As a nutrient-sensitive post-translational modification, 0-GlcNAcylation controls the intensity of signals travelling through different pathways according to the nutritional status of the cell (8). However, the major pathways controlling the 0-GIcNAcylation of eNOS in response to hypoglycemia, as well as the underlying mechanisms by which hypoglycemia changes eNOS activity, are unknown. One major biochemical pathway controlling hypoglycemia-induced O-GlcNAcylation have recently been shown: AMPK activation induced by hypoglycemia (9). Hypoglycemia-induced activation of OGT via AMPK, and the activation of the OGT by this mechanism, were found to increase protein O-GlcNAcylation $(9,10)$. We therefore hypothesized that hypoglycemia may affect eNOS activity via AMPK-induced OGT activation, resulting in O-GlcNAcylation and changes in NO production.

O-GlcNAcylation often competes with phosphorylation in the regulatory pathways of the cell, competition prediction server has identified several Ser and Thr residues in eNOS that may be targeted by phosphorylation or O-GIcNAcylation $(11,12)$. In eNOS, hyperglycemia increases O-GIcNAcylation and reciprocally decreases phosphorylation at Ser-1177 $(12,13)$. Previous investigations have mainly focused on how hyperglycemia induces an imbalance of competitive modification between 0-GIcNAcylation and phosphorylation in eNOS; however, few investigations have analyzed the effects of hypoglycemia on this competitive modification (14). The objectives of the present work were to elucidate the glycosylation of eNOS sites in response to hypoglycemia and to identify potential O-GlcNAcylation sites. To identify potential O-GICNAcylation sites, the glycosylated amino acid residues were identified by the fractionation of chymotryptic peptides using high-pressure liquid chromatography-mass spectrometry (HPLC-MS).

\section{Methods}

\section{Plasmid Construction}

eNOS-wild-type (WT) - The cDNA sequence of human eNOS (kindly provided by Dr. Yong Xia) was subcloned into pCDN3.1 (His-tagged). eNOS-S1177A, eNOS-T866A, eNOS-S867A and eNOS-S738A are point mutant of eNOS-WT were constructed by site-directed mutagenesis (Ser1177, Thr866, Ser867, Ser738 mutated to Ala). All mutations were performed by TransGen (TransGen Biotech, BeiJing, China) and were confirmed by direct sequencing.

\section{Cell Culture, Transfection, and Hypoglycemia Induction}

Bovine aortic endothelial cells (BAECs, passages 6-10) and human embryonic kidney (HEK293) cells were cultured in DMEM (Invitrogen, Carlsbad, CA, USA) supplemented with 10\% (v/v) FBS (Invitrogen) and 
$1 \%(\mathrm{v} / \mathrm{v}$ ) penicillin/streptomycin (PS) (Beyotime Biotechnology, JiangSu, China) in a humidified incubator at $37^{\circ} \mathrm{C}$ with $5 \% \mathrm{CO}_{2}$. For cell transfection, BAECs grown in 100-mm dishes were transfected with plasmids encoding eNOS-WT and eNOS mutants using Lipofectamine 3000 (Invitrogen) following the manufacturer's instructions and then analyzed $48 \mathrm{~h}$ after transfection. HEK293 cells in 6-well cell culture plates were transfected with plasmids encoding eNOS-WT or the eNOS mutants using Lipofectamine 2000 (Invitrogen) following the manufacturer's instructions and then analyzed $24 \mathrm{~h}$ after transfection. To induce hypoglycemia (LG), BAECs and HEK293 cells were cultured with medium containing $1 \mathrm{mmol} / \mathrm{L}$ glucose.

\section{AMPKa1 Gene Silencing in BAECs}

The sense (5'-GAUCCAUCAUAUAGCUCAAdTdT-3') and antisense (3'-UUGAGCUAUAUGAUGGAUCdTdT-5') Small interfering RNA (siRNA) strands of AMPKa1 were purchased from Biomic (Nanjing, China). siRNA oligonucleotides (75 nM) were delivered into cells using Opti-MEM (Invitrogen) and Lipofectamine 2000 according to the manufacturer's protocol. After transfection for $48 \mathrm{~h}$, cells were subjected to further experiments.

\section{Preparation of Protein Samples from Cells}

After culturing, cells were harvested by scraping. Harvested cells were washed three times with ice-cold PBS, resuspended in $100 \mu$ lysis buffer (Beyotime) containing protease inhibitor cocktail (Beyotime) and PUGNAc (Sigma Aldrich, St. Louis, MO, USA) in a clean Eppendorf tube, and kept on ice for $1 \mathrm{~h}$. The homogenate was then centrifuged at $13800 \times g$ and $4{ }^{\circ} \mathrm{C}$ for $15 \mathrm{~min}$ and the supernatant were recovered. The concentration of supernatant was determined by the Bradford protein assay.

\section{Preparation of Protein Samples from Rat Aortae}

Male and Female Sprague-Dawley rats approximately 6 to 8 weeks of age and 180 to $220 \mathrm{~g}$ weight were purchased from Experimental Animal Center of Chongqing Medical University. All animal procedures were carried out according to the guidelines of the China Animal Protection Law and were approved of by the Institutional Ethics Committee of Chongqing Medical University [Permit No. SCXK (Chongqing) 20070001] and the State Science and Technology Commission of China. Animals were housed individually and maintained on a $12 \mathrm{~h}$ light $/ 12 \mathrm{~h}$ dark schedule at $22-23^{\circ} \mathrm{C}$ with ad libitum access to food and water.

After overnight fasted, rats (200-250 g) were randomly divided into 4 groups (5 rats in each group): control group, hypoglycemia for 3, 6 and 9 h group. In hypoglycemia groups, rats were treated by glargine insulin (Lantus-Solostar, Paris, France) with subcutaneous injection $(200 \mathrm{~g} / \mathrm{U})$. In control group, rats were treated by PBS with subcutaneous injection $(200 \mathrm{~g} / 1 \mathrm{ml})$. Blood glucose was monitored every $30 \mathrm{~min}$ from 0 to 9 hours after insulin injection via tail prick, hypoglycemia was considered when glycemic $<3$ $\mathrm{mmol} / \mathrm{l}(14,15)$. Rats were anesthetized by $2 \%$ sodium pentobarbital solution with subcutaneous injection $(0.002 \mathrm{ml} / \mathrm{g})$. The thoracic aorta was quickly removed, and washed by ice cold PBS. After washing with ice-cold PBS, rat thoracic aortae were cut into pieces. Tissues were resuspended in lysis 
buffer containing protease inhibitor cocktail and PUGNAc in a clean Eppendorf tube. Tubes were placed on a cracker and the tissues were homogenized for $70 \mathrm{~min}$ at $4^{\circ} \mathrm{C}$. Lysate supernatants were collected by centrifugation at $13800 \times \mathrm{g}$ for $15 \mathrm{~min}$ at $4{ }^{\circ} \mathrm{C}$. The concentration of supernatant was determined by the Bradford protein assay.

\section{Immunoprecipitation}

For immunoprecipitation, aliquots of cell homogenates were incubated with polyclonal antibodies targeting OGT $(4 \mu \mathrm{g} / \mathrm{ml})$ (Abcam, Cambridge, UK) overnight at $4{ }^{\circ} \mathrm{C}$. Protein G-Sepharose beads (GE Healthcare, Chicago, IL, USA) were added to the supernatant and incubated for $3 \mathrm{~h}$. The beads were washed thoroughly with lysis buffer and then eluted by boiling for 3 min in SDS-PAGE sample buffer (Beyotime).

\section{eNOS Pull-down Assay}

In BAEC and rat thoracic aorta, eNOS was extracted from the lysate by affinity precipitation using 2',5'ADP Sepharose beads (GE Healthcare). Cell/tissue lysates were and mixed with prepared 2',5'-ADP Sepharose resins ( $50 \%$ slurry) at $4{ }^{\circ} \mathrm{C}$ for $2 \mathrm{~h}$ with gentle shaking. The mixture was then centrifuged at $13800 \times g$ for $1 \mathrm{~min}$. The supernatant was discarded, and the resins were washed with washing buffer (PBS supplemented with $500 \mathrm{mM} \mathrm{NaCl}$ ) three times. Bound proteins were eluted by boiling the resins in $50 \mu \mathrm{l}$ SDS-PAGE sample buffer for $10 \mathrm{~min}$. For transfected cells, eNOS was extracted by affinity precipitation after transfection using the His-tag protein purification Kit (Beyotime) per the manufacturer's protocol.

\section{Immunoblotting}

For western blotting, proteins were fractionated by SDS-PAGE and transferred to PVDF membranes (BioRad, Hercules, CA, USA). After blocking for $1.5 \mathrm{~h}$ in blocking buffer (20 mM Tris- $\mathrm{HCl}(\mathrm{pH} 7.5), 150 \mathrm{mM}$ $\mathrm{NaCl}, 5 \%$ skim milk powder, $0.1 \%(\mathrm{v} / \mathrm{v})$ Tween-20) at room temperature, the membranes were reacted with the appropriate primary antibodies (primary antibodies 1:2000; diluted in blocking buffer) at $4{ }^{\circ} \mathrm{C}$ overnight. Thereafter, membranes were thoroughly washed three times with TBST $(20 \mathrm{mM}$ Tris- $\mathrm{Cl}(\mathrm{pH}$ 7.5), $150 \mathrm{mM} \mathrm{NaCl}, 0.1 \%(\mathrm{v} / \mathrm{v})$ Tween-20) for $10 \mathrm{~min}$ per wash. Membranes were then incubated in 1:5000 diluted secondary antibody for $1.5 \mathrm{~h}$, thoroughly washed three times (10 min per wash) with TBST, and finally detected by chemiluminescence (Advansta Inc., San Jose, CA, USA).

Primary antibodies targeting phospho-eNOS Ser1177, phospho-eNOS Ser633, phospho-eNOS Thr495, and eNOS were purchased from Cell Signaling Technology (Danvers, MA, USA); O-GIcNAc antibody (RL2) was purchased from Thermo Fisher Scientific (Waltham, MA, USA). Antibodies targeting 0-GIcNAcase (OGA), Threonine, phospho-AMPK Thr172, and anti-AMPKa1 were purchased from Abcam (Cambridge, UK); $\beta$ actin antibody was purchased from Proteintech (Rosemont, IL, USA). Goat anti-mouse IgG and Goat antirabbit lgG were purchased from Santa Cruz Biotechnology (Dallas, TX, USA) and Proteintech, respectively.

\section{Preparation of eNOS for HPLC-MS}


eNOS was extracted by affinity precipitation from eNOS-WT-transfected HEK293 cells using the His-tag protein purification Kit. Affinity-precipitated eNOS was fractionated by SDS-PAGE, and the gel was stained with Coomassie brilliant blue. Stained eNOS was excised from the gel and stored in a clean Eppendorf tube after the excised gel was proven to contain eNOS by immunoblotting using the Micro Protein PAGE Recovery Kit (Sangon Biotechnology, Shanghai, China). Gels in the Eppendorf tubes were decolorized and swelled. After de-coloration and swelling, absolute acetonitrile was added to shrink and solidify the gel. At last, the acetonitrile was then absorbed and the gel was heat-dried. Dithiothreitol (DTT) solution were added to the tubes and mixed thoroughly, then incubated at $56^{\circ} \mathrm{C}$ for $1 \mathrm{~h}$; after incubation, the solution was discarded and the sample was heat-dried. Indole-3-acetic acid (IAA) solution was added, mixed well, and the mixture was incubated at room temperature for $30 \mathrm{~min}$; thereafter, the solution was discarded and DTT was added. The mixture was incubated at room temperature for $15 \mathrm{~min}$ for neutralize any remaining IAA; the solution was then discarded and the sample was heat-dried. Chymotrypsin was added to the sample at an enzyme/protein ratio of 1:20 in a reaction system containing $200 \mu 50 \mathrm{mmol} / \mathrm{L}$ ammonium bicarbonate solution, and incubated at $37^{\circ} \mathrm{C}$ for $14 \mathrm{~h}$. After the enzymatic digestion was completed, the enzymatic digestion solution was successively added with $1 \%$ TFA solution, $60 \%$ acetonitrile solution, $0.1 \%$ TFA solution, and absolute acetonitrile solution. The reaction was conducted at $37^{\circ} \mathrm{C}$ for $1 \mathrm{~h}$ respectively, and the reaction solution was combined.

\section{HPLC-MS for Enrichment of eNOS 0-Glycosylation}

Digested samples were dissolved in A solution (deionized $\mathrm{H}_{2} \mathrm{O}$ containing $0.1 \%$ formic acid) and centrifuged at $1000 \times g$ for $5 \mathrm{~min}$; the supernatants were then tested using the Thermo Orbitrap Lumos HPLC-MS system with a data collection time of $120 \mathrm{~min}$, spray voltage of $2.20 \mathrm{kV}$, capillary temperature of $320^{\circ} \mathrm{C}$, collision energy of $50 \%$, first-level mass range of collection of $300-1800 \mathrm{~m} / \mathrm{z}$, and second-level scanning range of 100-1400 m/z. Data from HPLC-MS for the enrichment of eNOS O-glycosylation were provided by the Beijing Proteome Research Center Tandem Mass Spectrometry (MS/MS) laboratory (China).

\section{Measurement of NO}

The concentration of NO released from BAECs in culture medium was measured in terms of the concentration of nitrate and nitrite using a modified Griess reaction method with the Total Nitric Oxide Assay Kit (Beyotime). Aorta blood samples from normal and hypoglycemic rats were collected using vacuum hemostix. Plasma was stored at $-80^{\circ} \mathrm{C}$ until assay. NO concentrations were determined using commercially available enzyme-linked immunosorbent assay (ELISA) kits (Nanjing Jiancheng Bioengineering Institute, Nanjing, China) per the manufacturer's protocol.

\section{eNOS Activity Assay}

eNOS activity was measured from the conversion rate of L-[14C] Arginine to L-[14C] Citrulline in cells. The conversion was monitored at a total volume of 300 ul buffer containing $50 \mathrm{mM}$ Tris- $\mathrm{HCl}, \mathrm{pH} 7.4,5 \mu \mathrm{M} \mathrm{L-}$ [14C] arginine (Moravek, Brea, CA, USA), $45 \mu \mathrm{M}$ L-arginine, $0.5 \mathrm{mM} \mathrm{NADPH}, 10 \mu \mathrm{M} \mathrm{BH} 4,10 \mu \mathrm{g} / \mathrm{ml}$ 
calmodulin, and $10 \mathrm{nM}$ eNOS. The reactions were initiated by adding L-[14C] arginine and terminated by stop buffer (20 mM HEPES, $2 \mathrm{mM}$ EDTA) after $1 \mathrm{~h}$ incubation at $37^{\circ} \mathrm{C}$. L-[14C] Citrulline was separated by passing the reaction mixture through a Dowex AG 50W-X8 ( $\mathrm{Na}^{+}$form) (Sigma) cation exchange columns and quantitated by liquid scintillation counting. N(gamma)-nitro-L-arginine methyl ester (L-NAME; $5 \mathrm{mM}$ ) inhibitory activity was analyzed to determine the concentration of L-[14C] citrulline converted by eNOS.

\section{Statistical analysis}

All data are presented as the mean \pm standard error of the mean (SEM). Statistical analysis was performed using SPSS by two-way analysis of variance (ANOVA), one-way ANOVA, or unpaired Student's $t$-test. A value of $P<0.05$ was considered statistically significant. Data analysis was performed using SPSS version 25 (SPSS Inc/IBM, Chicago, III, USA).

\section{Results}

\section{Hypoglycemia increases eNOS 0-GIcNAcylation and has no effect on O-linked phosphoserine (Ser1179)}

To confirm that hypoglycemia affects eNOS O-GIcNAcylation, western blotting was performed to detect 0GIcNAcylation and phospho-eNOS (Ser1179) under glucose deprivation. As demonstrated in Fig. 1A, the ratio of eNOS O-GIcNAcylation to eNOS increased by 3.5 -fold over $10 \mathrm{~h}$ of glucose deprivation, whereas phospho-eNOS (Ser1179) was not affected, suggesting that glucose deprivation only increased eNOS OGlcNAcylation. To determine the concentration of glucose with the same effect on eNOS as glucose deprivation, western blotting was performed to detect O-GIcNAcylation and phospho-eNOS (Ser1179) under different low-glucose concentrations. As demonstrated in Fig. 1B, the ratio of eNOS OGIcNAcylation to eNOS increased by 2.2-fold after $6 \mathrm{~h}$ of graded glucose concentration reduction from 5.5 to $1 \mathrm{mM}$; this ratio continued to increase from 2.2 to 2.5 -fold following a graded reduction from 1 to 0 $\mathrm{mM}$. In contrast, phospho-eNOS (Ser1179) was not changed, suggesting that $1 \mathrm{mM}$ glucose had the same effect on eNOS O-GIcNAcylation as glucose deprivation. As demonstrated in Fig. 1C, $1 \mathrm{mM}$ glucose had the same effect as glucose deprivation. These data strongly suggest that hypoglycemia only increases eNOS O-GIcNAcylation and has no effect on phospho-eNOS (Ser1179).

\section{Hypoglycemia increases eNOS O-GIcNAcylation through OGT up-regulation and AMPK activation}

Previous investigations demonstrated that OGT and OGA are two enzymes that regulate O-GIcNAcylation cycling (16). In order to identify which enzyme regulates eNOS O-GIcNAcylation under hypoglycemia, affinity precipitation of eNOS followed by western blotting with antibodies targeting OGT and OGA were performed, as shown in Fig. 2A. It was found that hypoglycemia increased OGT by $50 \%$, while reciprocal OGA expression was unchanged. It was observed that Thr phosphorylation could activate OGT (17); therefore, to confirm that hypoglycemia activates OGT via phosphorylation, the immunoprecipitation of OGT followed by western blotting with antibodies targeting phospho-Thr were performed. As demonstrated in Fig. 2B, the ratio of phospho-Thr OGT to OGT increased by $50 \%$ after incubation for $6 \mathrm{~h}$ under hypoglycemic conditions, suggesting that hypoglycemia increased OGT phosphorylation at its Thr 
site. These data suggest that hypoglycemia not only increases eNOS O-GIcNAcylation by inducing the binding of OGT to eNOS, but also activates OGT through its phosphorylation. As a cell energy sensor, AMPK can be activated by a decreased extracellular ATP/ADP ratio and plays a role in protein 0GlcNAcylation $(9,17)$. To confirm that AMPK played a role in eNOS O-GIcNAcylation, western blotting to detect O-GIcNAcylation, eNOS, P-AMPK Thr172, and AMPK was performed. As demonstrated in Fig. 2C, the ratio of eNOS O-GIcNAcylation to eNOS increased by 2.5-fold, while the ratio of P-AMPK to AMPK was also increased by 2 -fold, after $6 \mathrm{~h}$ incubation under hypoglycemic conditions. AMPKa1 knockdown was shown to reverse these effects of eNOS O-GIcNAcylation and AMPK phosphorylation. These data strongly suggest that hypoglycemia increases eNOS O-GIcNAcylation in an AMPK-dependent manner.

\section{Hypoglycemia increases eNOS 0-GIcNAcylation in rat aortae}

In the above results, we identified that hypoglycemia increases eNOS O-GIcNAcylation in vitro. To confirm that hypoglycemia also increases eNOS O-GIcNAcylation in vivo, we measured the blood glucose concentration of rats after insulin injection. As demonstrated in Fig. 3A, rats subcutaneously injected with insulin showed significantly lower rates of glycemia at $3 \mathrm{~h}$ after insulin injection. Western blotting to detect O-GIcNAcylated and phospho-eNOS (Ser1177) under hypoglycemia were performed. As demonstrated in Fig. 3B, the ratio of eNOS O-GIcNAcylation to eNOS increased by 2.5 -fold over $9 \mathrm{~h}$ following hypoglycemia exposure, whereas phospho-eNOS (Ser1177) was not changed. These data strongly suggest that hypoglycemia only increases eNOS O-GIcNAcylation and has no effect on phosphoeNOS (Ser1177) both in vitro and in vivo.

\section{Hypoglycemia increases 0-GIcNAcylation at the mutated eNOS Akt phosphorylation site}

To confirm whether eNOS has a novel O-GIcNAcylation sites, the effects of hypoglycemia on eNOS OGIcNAcylation and O-linked phosphoserine at this residue were evaluated using WT-eNOS and S1177AeNOS. As demonstrated in Fig. 4A, hypoglycemia increased the O-GlcNAcylation of WT-eNOS and had no effect on phospho-eNOS (Ser1177). As demonstrated in Fig. 4B, S1177A-eNOS stills exists 0GlcNAcylation. As demonstrated in Fig. 4C, O-GlcNAcylation was increased in S1177A-eNOS in response to hypoglycemia. As demonstrated in Fig. 4D, BAECs transfected with WT-eNOS and S1177-eNOS both showed an increase in 0-GIcNAcylation in response to hypoglycemia. These data suggest that eNOS may have a novel O-GIcNAcylation sites that is activated in response to hypoglycemia.

\section{HPLC-MS and MS/MS lon search for the hypoglycemia-dependent O-GICNAcylation sites of eNOS}

To determine whether eNOS has novel O-GICNAcylation sites, purified eNOS samples (excised gel) were analyzed by HPLC-MS, as demonstrated in Fig. 5A. To confirm that the analysis samples for HPLC-MS contained eNOS, proteins were recovered from the gels using the Micro Protein PAGE Recovery Kit. Western blotting against eNOS was then performed, as shown in in Fig. 5A (inset). To determine whether the novel O-GlcNAcylation sites obtained by MS/MS Ion Search (Fig. 5B and 5C (left panel)) were in fact major hypoglycemia-dependent O-GIcNAcylation sites, western blotting to detect O-GlcNAcylation under hypoglycemia was performed. As demonstrated in Fig. 5B (right panel), HEK293 cells transfected with 
S867A-eNOS showed an increase in 0-GlcNAcylation in response to hypoglycemia. As demonstrated in Fig. 5C (right panel), HEK293 cells transfected with T866A-eNOS showed no changes in 0-GlcNAcylation in response to hypoglycemia. Finally, as demonstrated in Fig. 5D, BAECs transfected with T866A-eNOS showed no changes in O-linked GIcNAc in response to hypoglycemia. These data strongly suggest that Thr866 is a novel O-linked GlcNAc site under hypoglycemia.

\section{Hypoglycemia increases eNOS activity}

To confirm that increased O-GICNAcylation would affect the synthesis of NO by eNOS under hypoglycemia, we measured changes in the production of NO in cell culture medium under hypoglycemic conditions. As demonstrated in Fig. 6A, the production of NO was increased by 3-fold after incubation for $10 \mathrm{~h}$ under hypoglycemic conditions. Moreover, to confirm that increased O-GlcNAcylation also affected eNOS function in vivo, we measured changes in the production of $\mathrm{NO}$ in rat aorta plasma. As demonstrated in Fig. 6B, the production of NO was increased by $40 \%$ at $6 \mathrm{~h}$ after hypoglycemia induction. These data strongly suggest that hypoglycemia increases the NO synthesis function of eNOS both in vitro and in vivo. Above, we showed that eNOS has a novel hypoglycemia-dependent O-GIcNAcylation site. To confirm that the novel O-GIcNAcylation site would affect NO synthesis by eNOS, we analyzed the changes in eNOS activity in WT-eNOS and T866A-eNOS in transfected HEK293 cells. As demonstrated in Fig. 6C, eNOS -catalyzed L-[14C] citrulline formation by WT-eNOS was increased by 10 -fold compare to that in T866A-eNOS. These data show that eNOS has a novel hypoglycemia-dependent O-GlcNAcylation site, and that this site was located at Thr866.

\section{Discussion}

In this report, we have shown that hypoglycemia increases eNOS activity in cultured BAECs by increasing OGT phosphorylation and attachment to eNOS via AMPK activation, which increases eNOS modification by GICNAc. When incubated with $1 \mathrm{mM}$ glucose, AMPK knockdown by siRNA reversed these modifications, as expected $(9,17)$, suggesting that hypoglycemia-induced increases in 0-GIcNAcylation are required for energy sensors to sense a decrease in the ATP/ADP ratio. These modifications appear to occur specifically at the 0-GlcNAcylation site. Residues such as Ser1177 are modified by GlcNAc $(12,13)$; however, hypoglycemia did not decrease the rate of modification at these sites. In aortae from hypoglycemic rats, changes in both the covalent modification of eNOS and eNOS activity resembled those observed in BAECs cultured in $1 \mathrm{mM}$ glucose. To our knowledge, this is the first report showing eNOS modification by GICNAc under hypoglycemia, and the first to reveal its underlying mechanisms. Additionally, these findings are the first example of functional alterations in a cytoplasmic enzyme induced by these modifications under hypoglycemia. There exists previous studies demonstrated that cell proteins could dynamically modified by 0-GIcNAc moieties due to hypoglycemia $(9,10,18,19)$. Although these observations were not made in endothelial cells, but rather focused on modifications in whole cell proteins, the same mechanism most likely explains the increase in eNOS O-GIcNAcylation in hypoglycemia. 
These data are somewhat consistent with previous observations indicating that glucose deprivation increases protein O-GIcNAcylation through the up-regulation of $\operatorname{OGT}(10,17,19)$. However, detecting 0GlcNAcylation at the whole-cell protein level may have missed several crucial biological phenomena in response to hypoglycemia. Different post-translational modifications induce different responses to hypoglycemia, and competition has been observed among those modifications $(5,13)$. Because we investigated O-GIcNAcylation in eNOS isolated by affinity purification, the influence of the OGlcNAcylation of other proteins in response to hypoglycemia was not existed. Additionally, because we identified a competitive modification site of O-GIcNAcylation by immunoblotting, these findings may improve our understanding of how hypoglycemia regulates eNOS activity through post-translation modifications. In addition to the underlying mechanism of hyperglycemia-induced eNOS O-GIcNAcylation, hypoglycemia also increased OGT activity by inducing phosphorylation and attachment to eNOS in an AMPK-dependent manner. This indicates that hypoglycemia increases eNOS O-GIcNAcylation through another signal pathway that independent of increased HBP flux and appears distinct from previously reported hyperglycemia-induced O-GIcNAcylation (20). In the cardiovascular system of patients with diabetes, several pathophysiological changes occur to prevent the inhibition of eNOS activity by hyperglycemia (21). Epidemiological studies have established an association between inflammatory biomarkers and the occurrence of diabetes and related complications (22). Diabetes increases the serum levels of cytokines, inflammatory factors, and induces oxidative stress; these responses also inhibit eNOS activity by decreasing Ser1177 phosphorylation (23). Hence, we propose that even in a pre-diabetic state without macro- or micro-vascular complications, the homeostasis of endothelial cells and eNOS activity is impaired by dysregulated metabolism, increased oxidative stress, and the expression of inflammatory factors. As a nutrient-sensitive modification site, the increased O-GIcNAcylation of eNOS may be initially triggered by metabolic dysregulation and function in the metabolic memory of endothelial cells $(12,13$, 20). Additionally, changes in phosphorylation allow rapid integrative responses to stimuli; thus, Ser1177 phosphorylation may have been decreased during early pre-diabetic stages. Moreover, hypoglycemia increased vessel blood flow in normal rats, but decreased endothelium-dependent relaxation in diabetes patients $(5,6)$. To some extent, these contradictory results which occurred in hypoglycemia rats may also support our findings.

Previous investigations have shown the significance of hyperglycemia-induced O-GlcNAcylation of eNOS in the inhibition of its enzyme activity $(12,13,20)$ by increasing 0 -GIcNAcylation in eNOS, accompanied by a reciprocal decrease in O-linked serine phosphorylation at Ser1177 $(12,13)$. However, in this study, this reciprocal activity was not observed when hypoglycemia induced an increase in eNOS OGIcNAcylation both in vitro and in vivo. Because we determined that eNOS O-GIcNAcylation site under hypoglycemia condition is different from hyperglycemia. We demonstrated that our S1177A-eNOS mutant still underwent O-GIcNAcylation, and that hypoglycemia induced this modification. Based on these phenomena, we propose that eNOS contains a potential O-GIcNAcylation site that is modified in response to hypoglycemia. Through HPLC-MS, we identified novel hypoglycemia-dependent OGIcNAcylation sites in eNOS. We confirmed the identities of the novel O-GlcNAcylation sites by transfecting and expressing site-specific mutant eNOS proteins based on the sites predicted by MS. When 
transfected HEK293 cells and BAECs were incubated in hypoglycemic conditions, the 0-GlcNAcylation of T866A-eNOS was not increased, showing that Thr866 is a novel O-GlcNAcylation site. Moreover, compared to that of T866A-eNOS, the activity of WT-eNOS was increased 8- to 10-fold. These data suggest that the regulation of eNOS activity under hypoglycemia occurs in part through the regulation of O-GIcNAcylation. This is the first study to construct functional alterations in eNOS induced by novel OGlcNAcylation site modification. These data are partially consistent with previous results showing that hypoglycemia increases eNOS activity (5).

However, whether hypoglycemia increases NO production and blood flow in the hypothalamichypophyseal portal system by increasing Ser1177 eNOS phosphorylation is still controversial $(5,24)$. Based on our findings, we suspect that this occurs through increased 0-GlcNAcylation of eNOS Thr866. Furthermore, we suspect that this modification may activate enzyme activity by increasing the $\mathrm{Km}$ of the enzyme. In our experiment, we first purified eNOS from cultured cells by affinity precipitation to ensure that NO synthesized by cell components or tissues other than eNOS was eliminated. Moreover, we excluded other positive regulatory site of eNOS to ensure that eNOS activity was only related to its OGlcNAcylation. It is known that physiological concentrations of insulin may increase eNOS activity by increasing phosphorylation at Ser1177 (25). This effect of insulin was eliminated because cells were treated in "pure" hypoglycemic conditions without any eNOS activators. Hence, the only factor affecting post-translational modifications of eNOS was a decreased glucose concentration. This novel 0GIcNAcylation site may be a specific "metabolic memory point" for eNOS in response to hypoglycemia. Furthermore, the novel modification reported here may provide a basis for the development of new energy-sensitive targets to prevent obesity-induced angiosclerosis. Such a target may help to prevent the development and progression of diabetes-associated severe vasospasm.

There were several limitations to this study. Acetylation is another nutrient-sensitive post-translational modification of eNOS, and its effect on eNOS activity in hypoglycemic conditions must be clarified in subsequent studies. In the future, the effect of the T866A eNOS mutant on endothelial cell signaling transduction and its protein-protein interaction should be investigated.

\section{Conclusions}

Our study demonstrates that hypoglycemia increases eNOS O-GIcNAcylation in vivo and in vitro. Hypoglycemia induced O-GIcNAcylation activation, possibly via AMPK-OGT pathway, hence pointing to the Thr866 as a novel O-GlcNAcylation site involved in hypoglycemia-mediated NO synthesis.

\section{Abbreviations}

eNOS

endothelial nitric oxide synthase

\section{BAECs}

bovine aortic endothelial cells 
OGT

O-GIcNAc transferase

\section{AMPK}

AMP kinase

NO

nitric oxide

HPLC-MS

high-pressure liquid chromatography-mass spectrometry

SiRNA

Small interfering RNA (siRNA)

\section{Declarations}

\section{Ethics approval and consent to participate}

All animal procedures were carried out according to the guidelines of the China Animal Protection Law and were approved of by the Institutional Ethics Committee of Chongqing Medical University [Permit No. SCXK (Chongqing) 2007-0001] and the State Science and Technology Commission of China.

\section{Consent for publication}

Not applicable

\section{Availability of data and materials}

The datasets used and analysed during the current study are available from the corresponding author on reasonable request.

All data generated or analysed during this study are included in this published article and its supplementary information files.

\section{Competing interests}

The authors declare that they have no competing interests

\section{Funding}

This work was supported by grants from the National Basic Research program of China (No.2014CB542402) and the National Nature Science Foundation of China (No.81270210).

\section{Authors' contributions}

An He, analyzed, collected the data and wrote the manuscript. Suxin Luo and Piqiang Zhong ensure the English language is of sufficient quality to be understood and fund the study. An He, Yang Long and 
Shupeng Hu isolated and purified the BAECs from bovine thoracic aorta. Yong Xia conceived, designed and fund the study, and conducted the experiments. All authors read and approved the final manuscript.

\section{Acknowledgements}

Not applicable

\section{References}

1. Erkens R, Suvorava T, Kramer CM, Diederich LD, Kelm M, Cortese-Krott MM.. (2017). Modulation of local and systemic heterocellular communication by mechanical forces: a role of endothelial nitric oxide synthase. Antioxidants \& Redox Signaling, ars.2016.6904.

2. Takemoto M, Egashira $K$, Usui M, Numaguchi $K$, Tomita $H$, Tsutsui $H$, et al. Important role of tissue angiotensin-converting enzyme activity in the pathogenesis of coronary vascular and myocardial structural changes induced by long-term blockade of nitric oxide synthesis in rats. Journal of Clinical Investigation. 1997;99(2):278-87.

3. Kader KN, Akella R, Ziats NP, Lakey LA, Harasaki H, Ranieri JP, et al. Enos-overexpressing endothelial cells inhibit platelet aggregation and smooth muscle cell proliferation in vitro. Tissue Eng. 2000;6(3):241.

4. Radomski MW, Vallance P, Whitley G, Foxwell N, Moncada S. Platelet adhesion to human vascular endothelium is modulated by constitutive and cytokine induced nitric oxide. Cardiovasc Res. 1993;27(7):1380.

5. Cabou C, Cani PD, Campistron G, Knauf C, Mathieu C, Sartori C, et al. Central insulin regulates heart rate and arterial blood flow: an endothelial nitric oxide synthase dependent mechanism altered during diabetes. Diabetes. 2007;56(12):2872-7.

6. Ceriello A, Novials A, Ortega E, Sala LL, Pujadas G, Testa R, et al. Evidence that hyperglycemia after recovery from hypoglycemia worsens endothelial function and increases oxidative stress and inflammation in healthy control subjects and subjects with type 1 diabetes. Diabetes. 2012;61(11):2993-7.

7. Dirsch VM, Heiss EH.. (2014). Regulation of enos enzyme activity by posttranslational modification. current pharmaceutical design, 20(22).

8. Issad T, Masson E, Pagesy P. O-glcnac modification, insulin signaling and diabetic complications. Diabetes Metabolism. 2010;36(6-part-P1):0-435.

9. Cheung WD, Hart GW. Amp-activated protein kinase and p38 mapk activate o-glcnacylation of neuronal proteins during glucose deprivation. J Biol Chem. 2008;283(19):13009-20.

10. Taylor RP, Parker GJ, Hazel MW, Soesanto Y, Fuller W, Yazzie MJ, et al. Glucose deprivation stimulates o-glcnac modification of proteins through up-regulation of o-linked nacetylglucosaminyltransferase. J Biol Chem. 2008;283(10):6050-7. 
11. Julenius K, Mølgaard A, Gupta R, Brunak S. Prediction, conservation analysis, and structural characterization of mammalian mucin-type o-glycosylation sites. Glycobiology. 2005;15(2):153-64.

12. Musicki B, Kramer MF, Becker RE, Burnett AL.. (2005). Inactivation of phosphorylated endothelial nitric oxide synthase (ser-1177) by o-glcnac in diabetes-associated erectile dysfunction. Proceedings of the National Academy of Sciences, 102(33), 11870-11875.

13. Liang DX, Diane E, Stefanie D, Qida J, Chengyu S, Michael B. Hyperglycemia inhibits endothelial nitric oxide synthase activity by posttranslational modification at the akt site. Journal of Clinical Investigation. 2001;108(9):1341-8.

14. Moore H, Craft TKS, Grimaldi LM, Babic B, Brunelli SA, Vannucci SJ. Moderate recurrent hypoglycemia during early development leads to persistent changes in affective behavior in the rat. Brain Behav Immun. 2010;24(5):0-849.

15. None. Glucose concentrations of less than $3.0 ? \mathrm{mmol} / \mathrm{l}(54 ? \mathrm{mg} / \mathrm{dl})$ should be reported in clinical trials: a joint position statement of the american diabetes association and the europian association for the study of diabetes. Diabetologia. 2017;60(1):3-6.

16. Aquino-Gil M, Pierce A, Perez-Cervera Y, Zenteno E, Lefebvre T. Ogt: a short overview of an enzyme standing out from usual glycosyltransferases. Biochem Soc Trans. 2017;45(2):365-70.

17. Bullen JW, Balsbaugh JL, Chanda D, Shabanowitz J, Hunt DF, Neumann D, et al. Cross-talk between two essential nutrient-sensitive enzymes: o-glcnac transferase (ogt) and amp-activated protein kinase (ampk). J Biol Chem. 2014;289(15):10592-606.

18. Kang JG, Park SY, Ji S, Jang I, Park S, Kim HS, et al. O-glcnac protein modification in cancer cells increases in response to glucose deprivation through glycogen degradation. J Biol Chem. 2009;284(50):34777-84.

19. Taylor RP, Geisler TS, Chambers JH, Mcclain DA. Up-regulation of o-glcnac transferase with glucose deprivation in hepg2 cells is mediated by decreased hexosamine pathway flux. J Biol Chem. 2009;284(6):3425-32.

20. Issad T, Masson E, Pagesy P. O-glcnac modification, insulin signaling and diabetic complications. Diabetes Metabolism. 2010;36(1):6-part-P. 0-435.

21. Shi Y, Vanhoutte PM. Macro- and microvascular endothelial dysfunction in diabetes. Journal of Diabetes. 2017;9(5):434-49.

22. Lontchiyimagou E, Sobngwi E, Matsha TE, Kengne AP. Diabetes mellitus and inflammation. Curr DiabRep. 2013;13(3):435-44.

23. Musicki B, Burnett AL. Endothelial dysfunction in diabetic erectile dysfunction. Int J Impot Res. 2007;19(2):129-38.

24. Fioramonti X, Marsollier N, Song Z, Fakira KA, Patel RM, Brown S, et al. Ventromedial hypothalamic nitric oxide production is necessary for hypoglycemia detection and counterregulation. Diabetes. 2010;59(2):519-28.

25. Kuboki K, et al. Regulation of endothelial constitutive nitric oxide synthase gene expression in endothelial cells and in vivo. Circulation. 2000;101:676-81. 
A
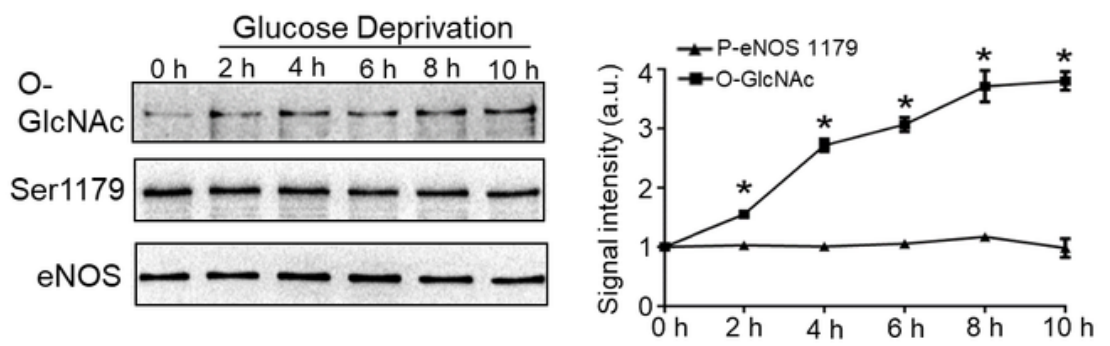

B



C
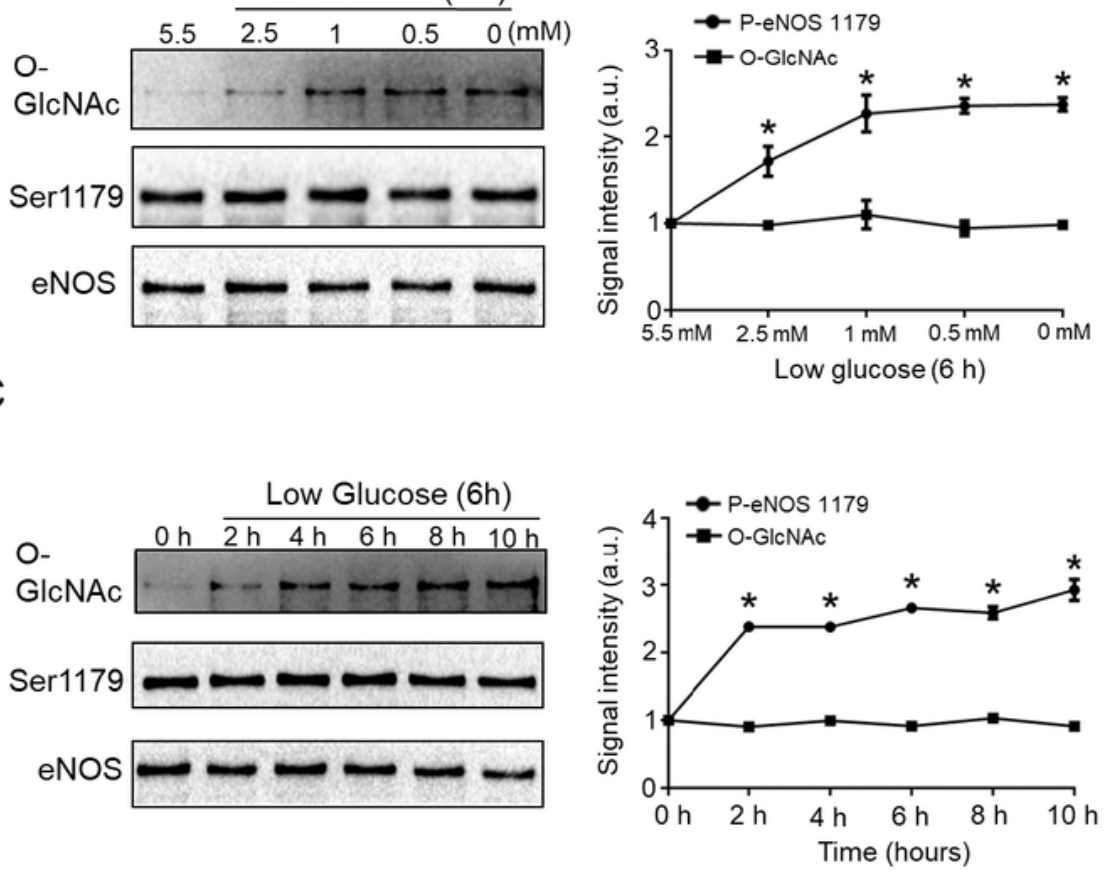

Figure 1

Figure 2

Effect of hypoglycemia on bovine aortic endothelial cell (BAEC) endothelial nitric oxide synthase (eNOS) O-GIcNAcylation. A: Left panel: Representative western blot of BAEC eNOS O-GIcNAcylation and phosphoeNOS (Ser1177) from control or glucose deprivation-treated cells $(n=3)$ over 10 h. Right panel: 
quantification of the ratio between O-GIcNAcylation, phosphorylated eNOS (P-eNOS), and eNOS. B: Left panel: representative western blot of BAEC O-GIcNAcylation, P-eNOS, and eNOS in response to a decrease in extracellular glucose from 5.5 to $0 \mathrm{mM}(\mathrm{n}=3)$ over $6 \mathrm{~h}$. Right panel: quantification of the ratio between O-GIcNAcylation, P-eNOS, and eNOS. C: Left panel: representative western blot of BAEC eNOS OGlcNAcylation and phospho-eNOS (Ser1177) from control or $1 \mathrm{mM}$ glucose-treated cells $(n=3)$ over $10 \mathrm{~h}$. Right panel: quantification of the ratio between O-GIcNAcylation, P-eNOS, and eNOS. Data are shown as the mean \pm SEM and represented as a percentage of the control, where the control group was considered to be $100 \% .{ }^{*} \mathrm{P}<0.05$ vs. control. Squares $=0-$ GlcNAc; circles $=\mathrm{P}-\mathrm{eNOS} 1179$. 
A
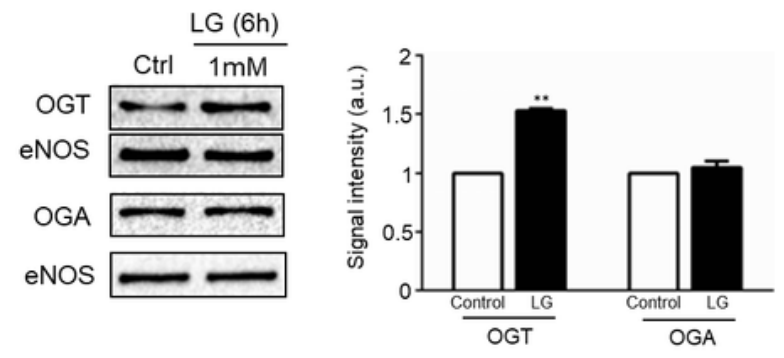

B
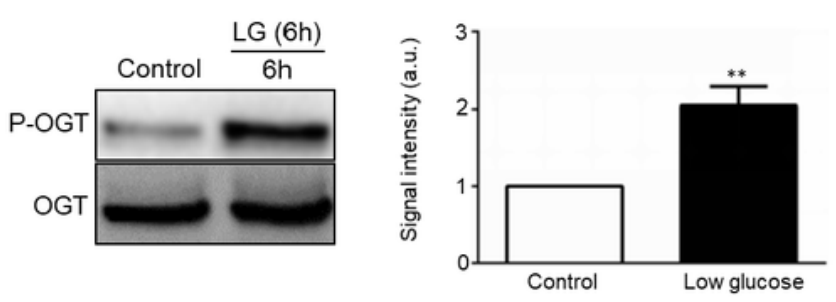

C
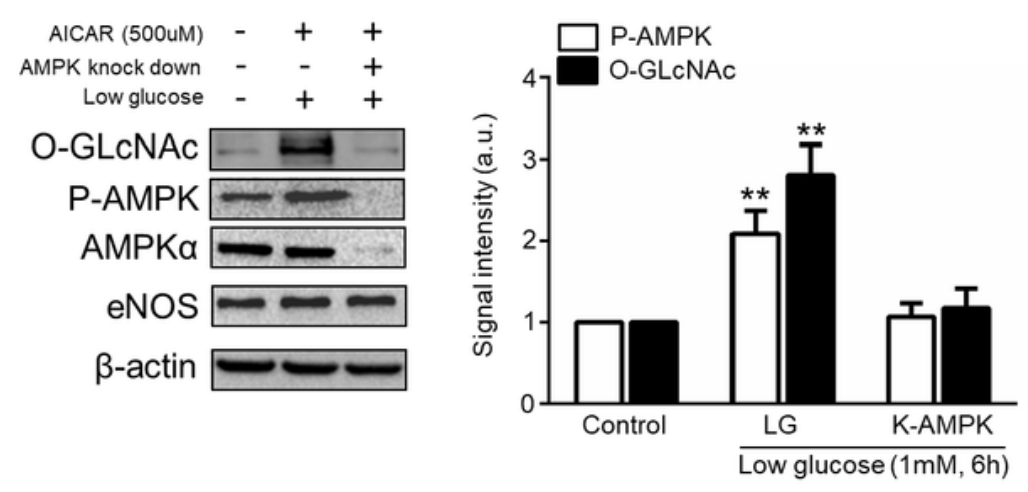

Figure 2

\section{Figure 4}

Increased O-GIcNAcylation stimulated by hypoglycemia is caused by AMP kinase (AMPK)-dependent OGIcNAc transferase (OGT) activation A: Left panel: representative western blot of OGT and O-GIcNAcase (OGA) from control or hypoglycemia (LG)-treated bovine aortic endothelial cells (BAECs) $(n=3)$ incubated for $6 \mathrm{~h}$. Right panel: quantification of the ratio between OGT and OGA. B: Left panel: representative western blot of phospho-threonine OGT and OGT from control or LG-treated BAECs $(n=3)$ incubated for 6 
h. Right panel: quantification of the ratio between phospho-threonine OGT and OGT. C: Left panel: representative western blot of AMPK, P-AMPK, eNOS O-GIcNAcylation, and eNOS from control or hypoglycemia-treated BAECs or AMPKa1-knockdown BAECs $(n=3)$ incubated for $6 \mathrm{~h}$. Right panel: quantification of the ratio of AMPK, P-AMPK, eNOS O-GIcNAcylation, and eNOS. Data are shown as the mean \pm SEM and represented as a percentage of control, where the control group was considered to be $100 \%$. $* *$ P $<0.01$ vs. control. White $=$ P-AMPK; black $=0-$ GlcNAc.

A

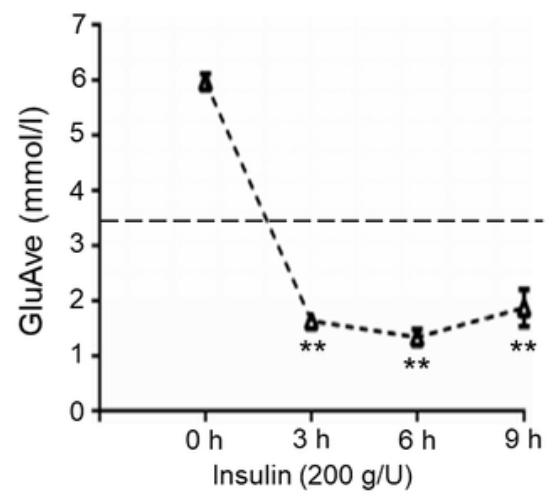

B



Figure 3

Figure 6 
Effect of hypoglycemia on BAEC eNOS O-GlcNAcylation in rat aortae. A: Blood glucose levels in response to insulin-induced hypoglycemia ( $200 \mathrm{~g} / \mathrm{U}$, subcutaneous) in a rat model $(n=5)$. B: Left panel:

representative western blot of O-linked GIc-NAc, P-eNOS and eNOS from the thoracic aorta of control or insulin-treated rats injected subcutaneously with insulin $(n=5)$ at 3-9 h after injection. Right panel:

quantification of the ratio between O-GIcNAcylation, P-eNOS, and eNOS. Data are shown as the mean \pm SEM and represented as a percentage of control, where the control group was considered to be $100 \%$. * $\mathrm{P}<0.01$ vs. control. White $=$ control; black $=$ hypoglycemia

A

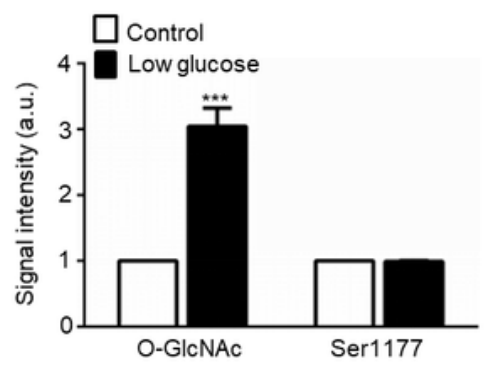

B
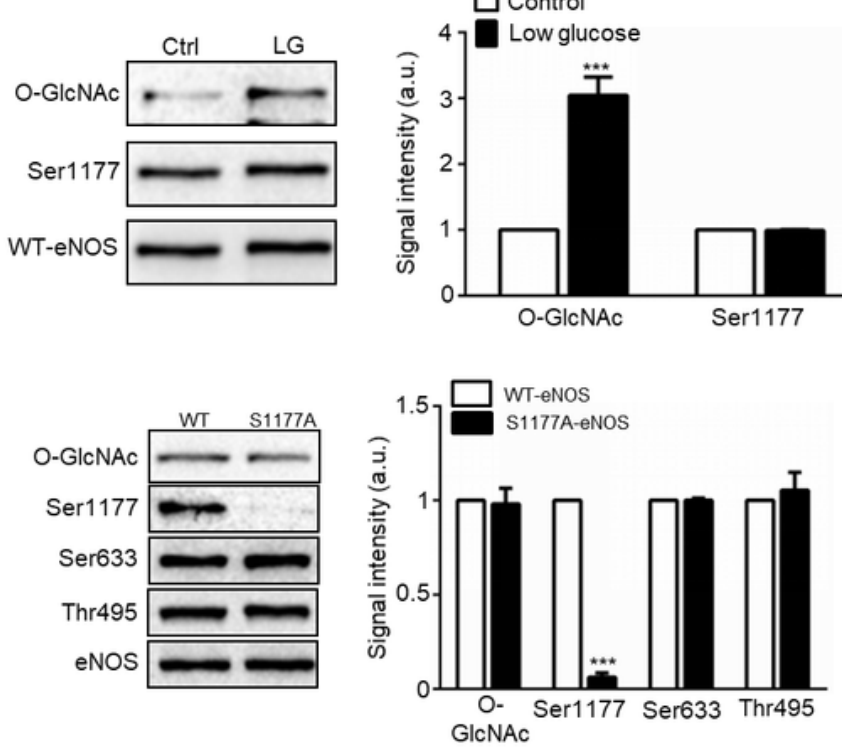

C
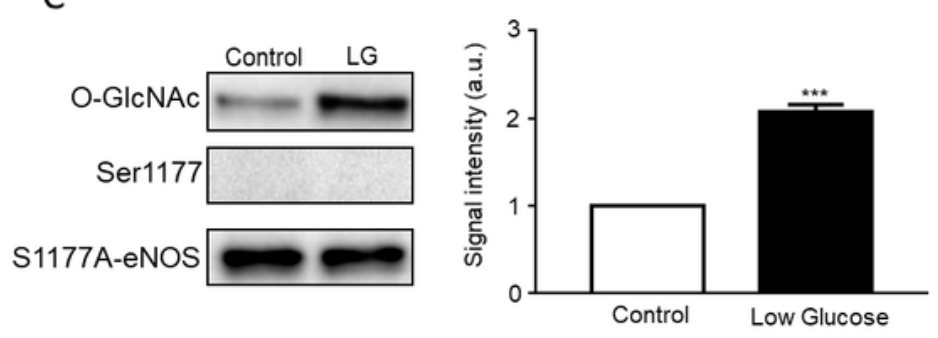

D
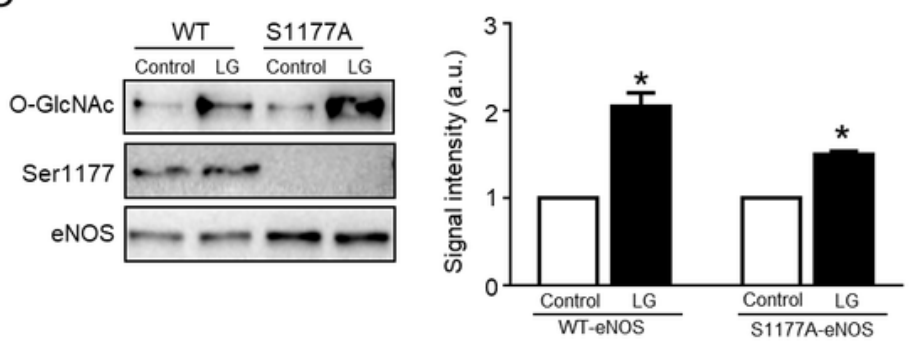

Figure 4 


\section{Figure 8}

Effect of hypoglycemia on 0-GlcNAcylation and phospho-eNOS (Ser1177) in His-tagged wild-type (WT)eNOS and S1177A-eNOS. A: Left panel: representative western blot of His-tagged WT-eNOS-transfected HEK293 cells, O-GlcNAcylation and phospho-eNOS (Ser1177) from control or hypoglycemia-treated cells $(n=3)$ incubated for $6 \mathrm{~h}$. Right panel: quantification of the ratio of O-GlcNAcylation and phospho-eNOS (Ser1177). B: Left panel: representative western blot of WT-eNOS- or S1177A-eNOS-transfected HEK293 cells showing O-GIcNAcylation, phospho-eNOS (Ser1177), phospho-eNOS (Thr495), and phospho-eNOS (Ser633) under normal culture conditions. Right panel: quantification of the ratio of O-linked GIcNAc, phospho-eNOS (Ser1177), phospho-eNOS (Thr495), and phospho-eNOS (Ser633). White = WT-eNOS; black = S1177A-eNOS C: Left panel: representative western blot of S1177A-eNOS-transfected HEK293 cells showing 0-GIcNAcylation and phospho-eNOS (Ser1177) in control or hypoglycemia-treated cells ( $\mathrm{n}=$ 3 ) incubated for $6 \mathrm{~h}$. Right panel: quantification of the ratio of O-GlcNAcylation and phospho-eNOS (Ser1177). D: Left panel: representative western blot of WT-eNOS- and S1177A-eNOS-transfected BAECs showing O-GIcNAcylation and phospho-eNOS (Ser1177) in control or hypoglycemia treated cells $(n=3)$ incubated for $6 \mathrm{~h}$. Right panel quantification of the ratio of 0-GlcNAcylation and phospho-eNOS (Ser1177). Data are shown as the mean \pm SEM and represented as a percentage of the control, where the

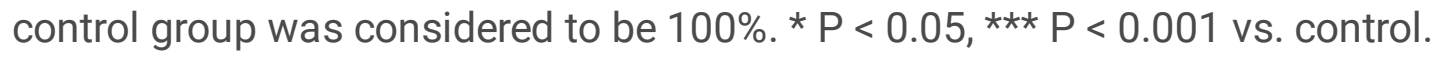


A

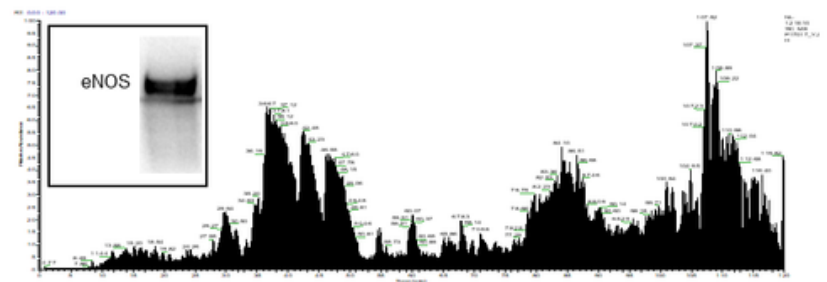

B
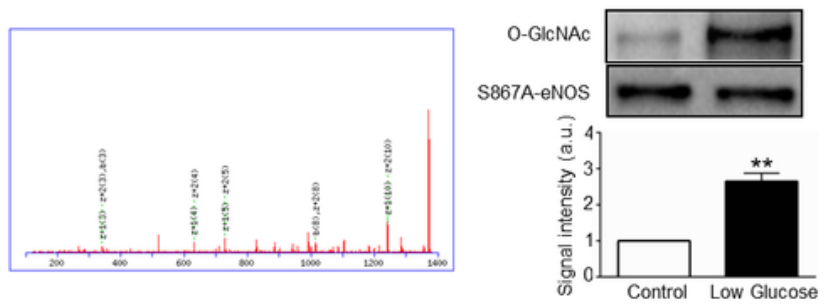

C
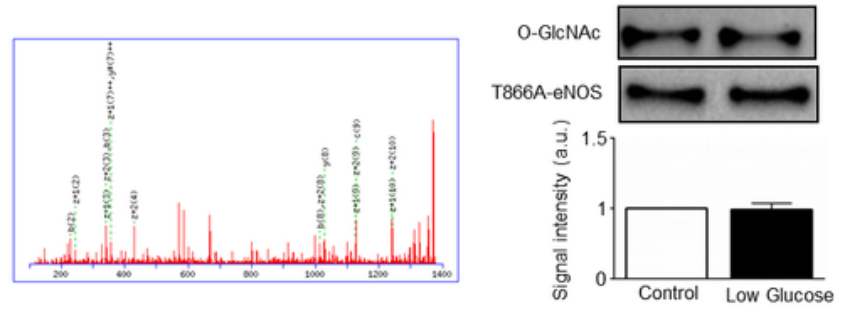

D
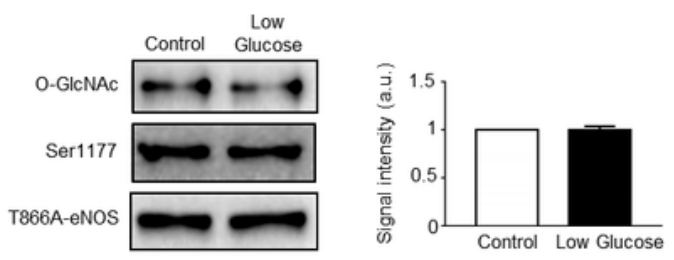

Figure 5

\section{Figure 10}

High-pressure liquid chromatography-mass spectrometry (HPLC-MS) and MS/MS lon Search for enriched eNOS O-GIcNAcylation sites. A: Inset, eNOS protein was recovered from gel. Fractionation by SDS-PAGE revealed a single major eNOS band, which was analyzed by HPLC-MS. B: Left panel: MS/MS lon Search score of eNOS Ser867 O-GIcNAcylation site. Upper right panel: representative western blot of S867AeNOS-transfected HEK293 cells showing O-linked GIcNAc from control or hypoglycemia-treated cells ( $\mathrm{n}=$ 
3) incubated for $6 \mathrm{~h}$. Lower right panel: quantification of the ratio between control and low glucose treated O-GIcNAcylation. C: Left panel: MS/MS Ion Search score of eNOS Thr866 O-GIcNAcylation site. Upper right panel: representative western blot of T866A-eNOS-transfected HEK293 cells showing O-linked GlcNAc from control or hypoglycemia-treated cells $(n=3)$ incubated for 6 h. Lower right panel: quantification of the ratio between control and low glucose treated O-GIcNAcylation. D: Left panel: representative western blot of T866A-eNOS-transfected BAECs showing 0-GIcNAcylation from control or hypoglycemia-treated cells $(n=3)$ incubated for $6 \mathrm{~h}$. Right panel: quantification of the ratio between control and low glucose treated O-GIcNAcylation. Data are shown as the mean \pm SEM and represented as a percentage of the control, where the control group was considered to be $100 \%$. ** $\mathrm{P}<0.01$ vs. control. White $=$ control; black $=$ low glucose (LG) . 
A

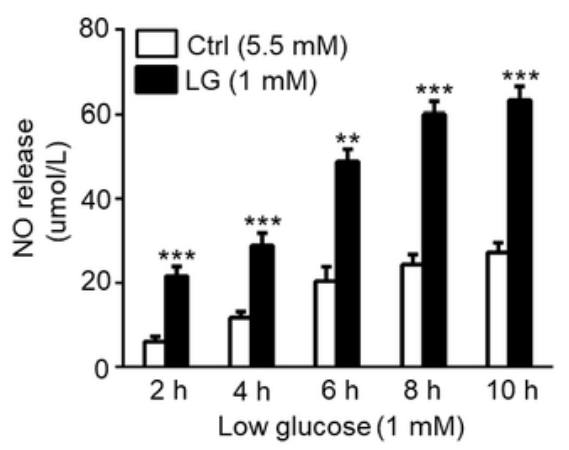

C
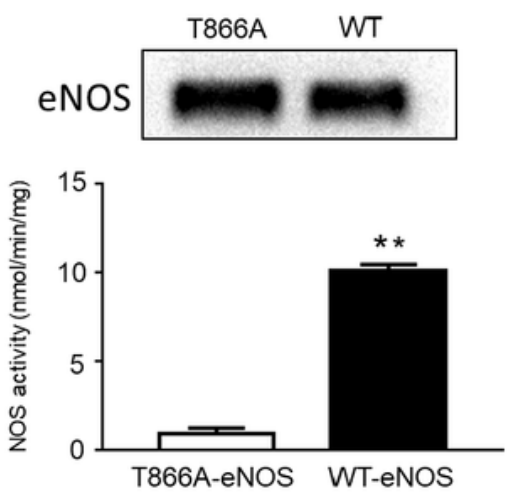

B

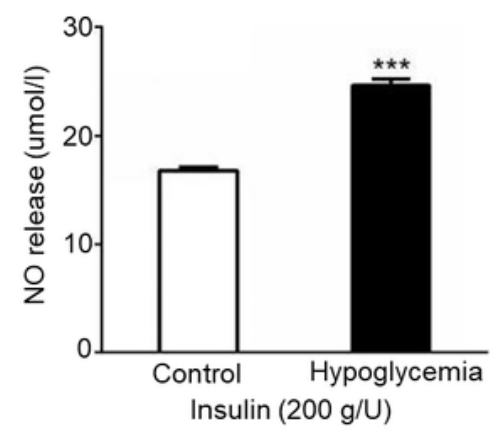

Figure 6

\section{Figure 12}

Effect of hypoglycemia on eNOS activity A: NO release in BAEC culture medium in control or hypoglycemia-treated BAECs $(n=3)$ over $10 \mathrm{~h}$. White = control; black = low glucose $(L G)$. B: NO release in rat aorta plasma from control or hypoglycemia-treated rat $(n=3)$ after $6 \mathrm{~h}$. White $=$ control; black $=$ hypoglycemia. C: Effect of hypoglycemia on L-[14C] citrulline in WT-eNOS- and T866A-eNOS-transfected HEK293 cells. Data are shown as the mean \pm SEM and represented as a percentage of the control, where 
the control group was considered to be $100 \%$. ${ }^{*} \mathrm{P}<0.05,{ }^{\star *} \mathrm{P}<0.01,{ }^{\star \star *} \mathrm{P}<0.001$ vs. control. White $=$ control; black = low glucose (LG). 